\title{
Are the effects of psychosocial exposures attributable to confounding? Evidence from a prospective observational study on psychological stress and mortality
}

\author{
J Macleod, G Davey Smith, P Heslop, C Metcalfe, D Carroll, C Hart
}

\begin{abstract}
Study objectives-To examine the association between perceived psychological stress and cause specific mortality in a population where perceived stress was not associated with material disadvantage.

Design-Prospective observational study with follow up of 21 years and repeat screening of half the cohort five years from baseline. Measures included perceived psychological stress, coronary risk factors, and indices of lifecourse socioeconomic position.
\end{abstract}

Setting-27 workplaces in Scotland.

Participants-5388 men (mean age 48 years) at first screening and 2595 men at second screening who had complete data on all measures.

Main outcome measures-Hazard ratios for all cause mortality and mortality from cardiovascular disease (ICD9 390-459), coronary heart disease (ICD9 410-414), smoking related cancers (ICD9 140, 141, 143-9, 150, 157, 160-163, 188 and 189), other cancers (ICD9 140-208 other than smoking related), stroke (ICD9 430-438), respiratory diseases (ICD9 460-519) and alcohol related causes (ICD9 141, 143-6, 148-9, 150, $155,161,291,303,571$ and 800-998).

Results-At first screening behavioural risk (higher smoking and alcohol consumption, lower exercise) was positively associated with stress. This relation was less apparent at second screening. Higher stress at first screening showed an apparent protective relation with all cause mortality and with most categories of cause specific mortality. In general, these estimates were attenuated on adjustment for social position. This pattern was also seen in relation to cumulative stress at first and second screening and with stress that increased between first and second screening. The pattern was most striking with regard to smoking related cancers: relative risk high compared with low stress at first screening, age adjusted 0.64 $(95 \%$ CI $0.42,0.96), p$ for trend 0.016 , fully adjusted $0.69(95 \%$ CI $0.45,1.06)$, p for trend 0.10; high compared with low cumulative stress, age adjusted 0.69 (95\% CI $0.44,1.09)$, $p$ for trend 0.12 , fully adjusted $0.76(95 \%$ CI $0.48,1.21)$, p for trend 0.25 ; increased compared with decreased stress, age adjusted 0.65 (95\% CI 0.40 ,
1.06), $p$ for trend 0.09 , fully adjusted 0.65 (95\% CI 0.40, 1.06), $\mathrm{p}$ for trend 0.08 .

Conclusions-This implausible protective relation between higher levels of stress, which were associated with increased smoking, and mortality from smoking related cancers, was probably a product of confounding. Plausible reported associations between psychosocial exposures and disease, in populations where such exposures are associated with material disadvantage, may be similarly produced by confounding, and of no causal significance. (F Epidemiol Community Health 2001;55:878-884)

Patterns of human health, for example social inequalities in health, are not fully explicable in terms of established physiological and behavioural disease risk factors. Psychosocial factors, such as stress and job control, are widely held to be important determinants of physical health. ${ }^{1-3}$ It has been suggested that such factors are important in the aetiology of socioeconomic health gradients and should be the target of interventions to reduce health inequalities. ${ }^{45}$ Studies on non-human primates have been cited as suggesting that psychoneuroendocrine mechanisms link psychosocial environment to physical disease ${ }^{6}{ }^{7}$ In addition, studies on human subjects indicate an association between adverse psychosocial exposure and unhealthy behaviour. ${ }^{89}$

Evidence for the importance of psychosocial factors in relation to population health is derived mainly from observational studies. Two recent reviews have interpreted this evidence as suggesting a causal relation between psychosocial exposures and coronary heart disease. ${ }^{23}$

Two general issues of observational epidemiology complicate interpretation of this evidence. Firstly, reporting bias is able to generate spurious exposure-outcome associations when both measurements depend on self report, and are inflated by a common reporting tendency. ${ }^{10}$ In many recent studies purporting to show a causal association between psychosocial exposures and health outcomes both have been measured via self report or have included a large subjective component. ${ }^{11-15}$ We have discussed this issue elsewhere. ${ }^{16}$

However, associations between psychosocial exposures and mortality are difficult to attribute to reporting bias. In most positive studies, heightened psychosocial risk was associated 
with adverse social position. ${ }^{17-20}$ Any measure associated with social disadvantage will also appear to be associated with poorer health as poorer health is associated with social disadvantage. Furthermore, socioeconomic position is often poorly measured in epidemiological studies and thus considerable residual confounding can exist despite apparent "adjustment" for social position in the analyses. ${ }^{21} 22$

The social patterning of many psychosocial constructs is likely to be influenced by discourse patterns in a particular society at a particular time. ${ }^{23}$ Thus, the social patterning of apparent psychosocial adversity, in terms of these exposures, may vary. If a psychosocial exposure has a true causal relation with health, then effects would be expected whatever the social distribution of this exposure. However if "effects" are principally the product of confounding they will only be seen when psychosocial adversity coincides with social disadvantage. Similarly, effects of psychosocial exposures seen in populations where heightened exposure is not associated with social disadvantage are difficult to attribute to confounding.

We examined the relation between perceived stress and mortality within a workplace recruited cohort of 5388 middle aged Scottish men followed up for 21 years. Approximately half of these men were re-examined five years after initial screening. This gave the opportunity to assess both the effect of stress measured on a single occasion (as previous studies have done) and the effect of stable or changing stress. We have previously reported how perceived stress within this cohort was not related to social disadvantage. ${ }^{24}$ Any relation between higher stress and poorer health in this population would therefore be unlikely to be attributable to socioeconomic confounding. Additionally we have reported a positive association between higher stress and unhealthy behaviour in this cohort, suggesting a mechanism through which stress could influence health. ${ }^{24}$ To establish whether stress did influence health we examined the relation between different categories of perceived stress and mortality. We also explored how this relation was influenced by social position, established disease risk factors, and by reporting bias.

\section{Methods}

This investigation is based on a cohort of men recruited from 27 workplaces in Scotland between 1970 and 1973. Seventy per cent of those invited completed a detailed questionnaire and attended for examination. The final achieved sample was of 5766 men aged 35-64 (mean age at first screening 48) of whom 2655 were re-screened in 1977 (mean screening interval 5.2 years).

Perceived stress was measured using the Reeder Stress Inventory (RSI). ${ }^{25}$ The Inventory contains four statements:

- In general I am usually tense or nervous

- There is a great amount of nervous strain connected with my daily activities
- At the end of the day I am completely exhausted mentally and physically

- My daily activities are extremely trying and stressful

Participants indicate the extent to which each statement applies to them using a four point Likert format. In the current cohort, internal consistency was high (Cronbach's $\alpha$ 0.78 at first screening, 0.80 at second screening). Repeatability was acceptable in those screened twice ( $\kappa$ was 0.28 unweighted, 0.45 weighted).

Principal components analysis and maximum likelihood factor analysis yielded a one factor solution. A scoring system was used to derive a summary score ranging from 1 (low perceived stress) to 8 (high perceived stress). ${ }^{25}$ Validity of the RSI has previously been suggested by a high degree of correlation with a Dutch stress scale devised by Dirken. ${ }^{26}$ In the Framingham study RSI stress was significantly correlated with all other psychosocial measures used, most markedly with Type A behaviour, tension, anger and emotional lability. ${ }^{27}$ Higher RSI stress was associated with increased use of tranquilisers in the Los Angeles Heart Study. ${ }^{28}$ This, along with the association between higher stress, increased smoking and increased alcohol consumption in the present study suggest, construct validity. ${ }^{24}$

Blood pressure, lung function, height and weight and body mass index (BMI) were measured in standard ways. ${ }^{29}$ At first screening, blood samples were taken for measurement of plasma cholesterol concentration. The self completed questionnaire collected information on physical exercise (hours per week), cigarette smoking (ex, current or never smokers; cigarettes smoked daily; age at initiation of smoking, inhalation) and consumption of alcohol. Own occupational social class at time of screening and at entry into the workforce, along with father's social class, were assigned on the basis of the registrar general classification. ${ }^{30}$ Participants were also asked whether they owned a car. An area-based measure of deprivation, derived according to the method of Carstairs and Morris, ${ }^{31}$ was assigned according to postcode of participants' home address.

In an attempt to quantify tendency to over-report physical symptoms we took five symptoms (polydipsia, polyuria, pruritus, blurred vision and increased skin infections). Participants were asked to report experience of these in the past year. These symptoms showed no relation to mortality over 21 years. We constructed a reporting tendency variable (0-5) based on a count of reports of these. Known diabetics $(n=29$ at first screening and $n=23$ at second screening) were excluded on the assumption that their reporting of these hyperglycaemic symptoms was more likely to reflect their diabetes than any reporting tendency.

Altogether 5388 men at first screening and 2595 men at second screening had complete information on all measures; analyses are based on these men. A full description of the methods and procedures used has been published elsewhere. ${ }^{24} 29$ 
Men who died over the 21 years of follow up from first screening were identified through flagging at the NHS Central Registry in Edinburgh, which also provides death certificates coded according to the ninth revision of the International Classification of Diseases. In addition to all cause mortality several categories of cause specific mortality were examined. These included deaths from cardiovascular causes (ICD9 codes 390-459); coronary heart disease (ICD9 410-414); stroke (ICD9 430-438) and respiratory diseases (ICD9 460-519). Deaths from cancer were taken to be those covered by ICD9 codes 140-208. Smoking related cancers were examined separately and were taken to be covered by ICD 9 codes 140, 141, 143-9, 150, $157,160-163,188$ and 189, as described elsewhere. $^{32}$ Alcohol related mortality was also examined separately. Alcohol related deaths were taken to be those covered by ICD 9 codes $141,143-6,148-9,150,155,161,291,303$, 571 and $800-998$ as described elsewhere. ${ }^{33}$

The distribution of stress scores at first screening showed some clumping around middle values and was positively skewed. Scores were categorised as high (6-8), medium (4-5) and low (1-3). In men screened twice stress scores were summed. This composite score was normally distributed and was divided on the basis of tertiles into high (10-16), medium (7-9) and low (2-6). Finally the difference between stress scores at first and second screening was calculated to allow division of participants into those whose perceived stress increased, decreased or remained stable over the screening interval. Relative risks of all cause and cause specific mortality in relation to different stress exposure categorisations were then calculated.

Survival analysis was undertaken using the proportional hazards method of $\mathrm{Cox},{ }^{34}$ hazard ratios were taken to represent relative risk. These are presented adjusted for age, adjusted for age and measures of social position, adjusted for age, social position and risk factors and adjusted for age, social position risk factors and reporting tendency. For models including perceived stress at first screening only, covariates are as measured at first screening. For models including stress measured on two occasions, covariates are as measured at second screening. In all models follow up is censored at 21 years from first screening. All analyses were undertaken using the software package STATA $6.0 .^{35}$

\section{Results}

Perceived stress showed a graded association with participants' current occupational social class at both first and second screening (first screening mean stress score for social class I =

Table 1 Relations between perceived stress at first screening and coronary risk factors at first screening

\begin{tabular}{|c|c|c|c|c|}
\hline & \multicolumn{3}{|c|}{ Perceived stress } & \multirow[b]{2}{*}{$p$ for trend } \\
\hline & $\begin{array}{l}\text { Low } \\
n=1765\end{array}$ & $\begin{array}{l}\text { Medium } \\
n=2912\end{array}$ & $\begin{array}{l}\text { High } \\
n=711\end{array}$ & \\
\hline Proportion smoking $>20$ cigarettes daily $(\%)$ & 18 & 21 & 26 & $<0.001$ \\
\hline Proportion consuming $>15$ units of alcohol weekly (\%) & 27 & 29 & 29 & 0.020 \\
\hline Proportion taking $<3$ hours exercise weekly $(\%)$ & 19 & 19 & 26 & 0.005 \\
\hline Proportion with body mass index $>25 \mathrm{~kg} / \mathrm{m}^{2}(\%)$ & 53 & 50 & 46 & 0.004 \\
\hline Proportion with diastolic blood pressure $\geqslant 90 \mathrm{~mm} \mathrm{Hg} \mathrm{( \% )}$ & 26 & 25 & 23 & 0.10 \\
\hline Proportion with plasma cholesterol $>5.5 \mathrm{mmol} / 1(\%)$ & 60 & 62 & 60 & 0.66 \\
\hline Proportion with $\mathrm{FEV}_{1}<90 \%$ of predicted value (\%) & 40 & 37 & 37 & 0.58 \\
\hline
\end{tabular}

Standardisation by the direct method for age and occupational class.

Table 2 Relations between coronary risk factors at second screening and sum of perceived stress scores at first and second screening

\begin{tabular}{|c|c|c|c|c|}
\hline & \multicolumn{3}{|c|}{ Perceived stress } & \multirow[b]{2}{*}{$p$ for trend } \\
\hline & $\begin{array}{l}\text { Low } \\
n=895\end{array}$ & $\begin{array}{l}\text { Medium } \\
n=874\end{array}$ & $\begin{array}{l}\text { High } \\
n=826\end{array}$ & \\
\hline Proportion smoking $>20$ cigarettes daily $(\%)$ & 21 & 27 & 25 & 0.11 \\
\hline Proportion consuming $>15$ units of alcohol weekly (\%) & 35 & 34 & 32 & 0.47 \\
\hline Proportion taking $<3$ hours exercise weekly $(\%)$ & 29 & 26 & 24 & 0.37 \\
\hline Proportion with body mass index $>25 \mathrm{~kg} / \mathrm{m}^{2}(\%)$ & 59 & 58 & 51 & 0.006 \\
\hline Proportion with diastolic blood pressure $\geqslant 90 \mathrm{~mm} \mathrm{Hg}(\%)$ & 29 & 28 & 26 & 0.13 \\
\hline Proportion with $\mathrm{FEV}_{1}<90 \%$ of predicted value (\%) & 43 & 40 & 41 & 0.050 \\
\hline
\end{tabular}

Standardisation by the direct method for age and occupational class.

Table 3 Relations between coronary risk factors at second screening and change in perceived stress score between first and second screening

\begin{tabular}{|c|c|c|c|c|}
\hline & \multicolumn{3}{|c|}{ Perceived stress } & \multirow[b]{2}{*}{$p$ for trend } \\
\hline & $\begin{array}{l}\text { Decreased } \\
n=828\end{array}$ & $\begin{array}{l}\text { Stable } \\
n=1085\end{array}$ & $\begin{array}{l}\text { Increased } \\
n=682\end{array}$ & \\
\hline Proportion smoking $>20$ cigarettes daily $(\%)$ & 24 & 25 & 25 & 0.60 \\
\hline Proportion consuming >15 units of alcohol weekly (\%) & 31 & 33 & 35 & 0.17 \\
\hline Proportion taking $<3$ hours exercise weekly $(\%)$ & 27 & 28 & 23 & 0.13 \\
\hline Proportion with body mass index $>25 \mathrm{~kg} / \mathrm{m}^{2}(\%)$ & 57 & 55 & 57 & 0.76 \\
\hline Proportion with diastolic blood pressure $\geqslant 90 \mathrm{~mm} \mathrm{Hg}(\%)$ & 29 & 27 & 27 & 0.31 \\
\hline Proportion with $\mathrm{FEV}_{1}<90 \%$ of predicted value (\%) & 43 & 41 & 40 & 0.08 \\
\hline
\end{tabular}

Standardisation by the direct method for age and occupational class. 
Table 4 Relative risk of mortality (95\% CI) associated with perceived stress at first screening

\begin{tabular}{|c|c|c|c|c|c|c|}
\hline & $\begin{array}{l}\text { Perceived } \\
\text { stress }\end{array}$ & Adjusted for age & $\begin{array}{l}\text { Adjusted for age and } \\
\text { occupational class }\end{array}$ & $\begin{array}{l}\text { Adjusted for age and } \\
\text { all markers of social } \\
\text { position }\end{array}$ & $\begin{array}{l}\text { Adjusted, for age, } \\
\text { social position, and } \\
\text { risk factorst }\end{array}$ & $\begin{array}{l}\text { Adjusted for age, social } \\
\text { position, risk factors and } \\
\text { reporting tendency }\end{array}$ \\
\hline All cause & High & $0.88(0.75,1.03)$ & $0.95(0.81,1.12)$ & $0.95(0.81,1.12)$ & $0.94(0.80,1.11)$ & $0.97(0.83,1.15)$ \\
\hline \multirow[t]{2}{*}{ (1528 deaths) } & Medium & $0.85(0.76,0.94)$ & $0.93(0.83,1.04)$ & $0.94(0.84,1.05)$ & $0.93(0.83,1.05)$ & $0.95(0.84,1.06)$ \\
\hline & Low & 1.00 & 1.00 & 1.00 & 1.00 & 1.00 \\
\hline $\mathrm{p}$ for trend & & 0.020 & 0.34 & 0.38 & 0.33 & 0.55 \\
\hline All cardiovascular & High & $0.84(0.67,1.05)$ & $0.90(0.72,1.13)$ & $0.90(0.72,1.13)$ & $0.92(0.73,1.16)$ & $0.94(0.74,1.18)$ \\
\hline \multirow[t]{2}{*}{ (785 deaths) } & Medium & $0.83(0.71,0.97)$ & $0.91(0.78,1.06)$ & $0.92(0.79,1.07)$ & $0.92(0.79,1.08)$ & $0.93(0.79,1.09)$ \\
\hline & Low & 1.00 & 1.00 & 1.00 & 1.00 & 1.00 \\
\hline $\mathrm{p}$ for trend & & 0.037 & 0.26 & 0.28 & 0.36 & 0.44 \\
\hline Coronary heart disease & High & $0.86(0.67,1.12)$ & $0.93(0.71,1.21)$ & $0.93(0.71,1.21)$ & $0.96(0.74,1.25)$ & $0.97(0.74,1.27)$ \\
\hline \multirow[t]{2}{*}{ (590 deaths) } & Medium & $0.86(0.72,1.03)$ & $0.94(0.79,1.13)$ & $0.95(0.79,1.14)$ & $0.97(0.81,1.16)$ & $0.97(0.81,1.17)$ \\
\hline & Low & 1.00 & 1.00 & 1.00 & 1.00 & 1.00 \\
\hline $\mathrm{p}$ for trend & & 0.14 & 0.50 & 0.52 & 0.72 & 0.78 \\
\hline Smoking related cancers & High & $0.64(0.42,0.96)$ & $0.71(0.47,1.08)$ & $0.71(0.47,1.08)$ & $0.66(0.44,1.01)$ & $0.69(0.45,1.06)$ \\
\hline \multirow[t]{2}{*}{ (269 deaths) } & Medium & $0.79(0.61,1.02)$ & $0.91(0.70,1.18)$ & $0.91(0.70,1.19)$ & $0.89(0.69,1.16)$ & $0.90(0.69,1.18)$ \\
\hline & Low & 1.00 & 1.00 & 1.00 & 1.00 & 1.00 \\
\hline $\mathrm{p}$ for trend & & 0.016 & 0.13 & 0.13 & 0.06 & 0.10 \\
\hline Other cancers & High & $0.91(0.59,1.40)$ & $0.91(0.59,1.40)$ & $0.93(0.60,1.44)$ & $0.94(0.61,1.46)$ & $1.00(0.64,1.56)$ \\
\hline \multirow[t]{2}{*}{ (221 deaths) } & Medium & $0.93(0.70,1.25)$ & $0.93(0.69,1.25)$ & $0.94(0.70,1.27)$ & $0.94(0.70,1.27)$ & $0.96(0.71,1.30)$ \\
\hline & Low & 1.00 & 1.00 & 1.00 & 1.00 & 1.00 \\
\hline $\mathrm{p}$ for trend & & 0.61 & 0.60 & 0.70 & 0.73 & 0.93 \\
\hline Stroke & High & $0.85(0.49,1.48)$ & $0.90(0.52,1.58)$ & $0.92(0.52,1.60)$ & $0.94(0.54,1.65)$ & $0.98(0.55,1.72)$ \\
\hline \multirow[t]{2}{*}{ (122 deaths) } & Medium & $0.75(0.51,1.10)$ & $0.80(0.54,1.19)$ & $0.81(0.55,1.20)$ & $0.81(0.54,1.21)$ & $0.82(0.55,1.23)$ \\
\hline & Low & 1.00 & 1.00 & 1.00 & 1.00 & 1.00 \\
\hline $\mathrm{p}$ for trend & & 0.32 & 0.51 & 0.54 & 0.59 & 0.68 \\
\hline Alcohol related & High & $1.13(0.63,2.01)$ & $1.29(0.72,2.31)$ & $1.26(0.71,2.26)$ & $1.22(0.68,2.20)$ & $1.30(0.72,2.35)$ \\
\hline \multirow{2}{*}{ (105 deaths) } & Medium & $0.86(0.56,1.32)$ & $1.01(0.65,1.56)$ & $1.01(0.65,1.55)$ & $0.97(0.63,1.51)$ & $1.00(0.64,1.54)$ \\
\hline & Low & 1.00 & 1.00 & 1.00 & 1.00 & 1.00 \\
\hline $\mathrm{p}$ for trend & & 0.91 & 0.51 & 0.54 & 0.59 & 0.68 \\
\hline Respiratory & High & $1.23(0.69,2.22)$ & $1.39(0.77,2.50)$ & $1.36(0.75,2.45)$ & $1.22(0.67,2.22)$ & $1.38(0.75,2.53)$ \\
\hline \multirow[t]{2}{*}{ (100 deaths) } & Medium & $0.94(0.61,1.46)$ & $1.09(0.69,1.71)$ & $1.11(0.71,1.74)$ & $0.96(0.61,1.52)$ & $0.99(0.63,1.56)$ \\
\hline & Low & 1.00 & 1.00 & 1.00 & 1.00 & 1.00 \\
\hline $\mathrm{p}$ for trend & & 0.62 & 0.31 & 0.33 & 0.61 & 0.40 \\
\hline
\end{tabular}

${ }^{\star}$ Current occupational class, occupational class of first employment, father's occupational class, car ownership, deprivation category of area of residence. + Cigarettes smoked daily, ex, current, never smokers, age at initiation of smoking, inhalation, alcohol consumption (0, $>0-15,>15$ units weekly), hours of exercise weekly, diastolic blood pressure $(\mathrm{mm} \mathrm{Hg})$, plasma cholesterol ( $\mathrm{mmol} / \mathrm{l})$, body mass index $\left(\mathrm{kg} / \mathrm{m}^{2}\right), \mathrm{FEV}_{1} \%$.

4.4 , social class $\mathrm{V}=2.8$, $\mathrm{p}$ for trend $<0.001$; second screening mean stress score social class $\mathrm{I}=4.5$, social class $\mathrm{V}=2.9 \mathrm{p}$ for trend $<0.001)$.

Table 1 shows the distribution of risk factors by stress score category at first screening. After standardisation for age and social class, greater stress at first screening is associated with an adverse pattern of behavioural risk (more smoking, less exercise, greater alcohol consumption) but shows no clear relation with physiological risk factors (blood pressure, blood cholesterol and lung function). Greater

Table 5 All cause and cause specific mortality according to the sum of perceived stress at first and second screening

\begin{tabular}{|c|c|c|c|c|c|}
\hline & Perceived stress & Adjusted for age & $\begin{array}{l}\text { Adjusted for age and } \\
\text { occupational class }\end{array}$ & $\begin{array}{l}\text { Adjusted, for age, } \\
\text { occupational class and risk } \\
\text { factors }^{\star}\end{array}$ & $\begin{array}{l}\text { Adjusted for age, } \\
\text { occupational class, risk } \\
\text { factors and reporting } \\
\text { tendency }\end{array}$ \\
\hline All cause & High & $0.92(0.76,1.11)$ & $1.00(0.83,1.23)$ & $0.98(0.81,1.19)$ & $1.00(0.82,1.22)$ \\
\hline \multirow{2}{*}{ (642 deaths) } & Medium & $0.93(0.77,1.11)$ & $1.00(0.82,1.20)$ & $0.98(0.81,1.19)$ & $0.99(0.82,1.20)$ \\
\hline & Low & 1.00 & 1.00 & 1.00 & 1.00 \\
\hline $\mathrm{p}$ for trend & & 0.38 & 0.93 & 0.85 & 0.98 \\
\hline All cardiovascular & High & $1.02(0.79,1.33)$ & $1.13(0.85,1.47)$ & $1.12(0.85,1.46)$ & $1.16(0.88,1.53)$ \\
\hline \multirow{2}{*}{ (335 deaths) } & Medium & $1.02(0.79,1.33)$ & $1.11(0.85,1.44)$ & $1.10(0.84,1.43)$ & $1.11(0.85,1.45)$ \\
\hline & Low & 1.00 & 1.00 & 1.00 & 1.00 \\
\hline $\mathrm{p}$ for trend & & 0.86 & 0.36 & 0.42 & 0.28 \\
\hline Coronary heart disease & High & $1.00(0.75,1.36)$ & $1.11(0.82,1.51)$ & $1.11(0.82,1.51)$ & $1.16(0.84,1.58)$ \\
\hline \multirow{2}{*}{ (258 deaths) } & Medium & $0.99(0.73,1.33)$ & $1.07(0.79,1.44)$ & $1.07(0.79,1.44)$ & $1.08(0.80,1.46)$ \\
\hline & Low & 1.00 & 1.00 & 1.00 & 1.00 \\
\hline $\mathrm{p}$ for trend & & 0.97 & 0.51 & 0.50 & 0.36 \\
\hline Smoking related cancers & High & $0.69(0.44,1.09)$ & $0.78(0.50,1.24)$ & $0.76(0.48,1.20)$ & $0.76(0.48,1.21)$ \\
\hline \multirow{2}{*}{$\begin{array}{l}\text { Smoking related cancers } \\
\text { (126 deaths) }\end{array}$} & Medium & $0.91(0.61,1.37)$ & $1.00(0.67,1.52)$ & $0.99(0.65,1.50)$ & $0.97(0.64,1.47)$ \\
\hline & Low & 1.00 & 1.00 & 1.00 & 1.00 \\
\hline $\mathrm{p}$ for trend & & 0.12 & 0.32 & 0.26 & 0.25 \\
\hline & High & $0.90(0.53,1.52)$ & $0.90(0.53,1.55)$ & $0.90(0.52,1.54)$ & $0.91(0.52,1.57)$ \\
\hline \multirow{2}{*}{$\begin{array}{l}\text { ( } 89 \text { deaths) } \\
\text { ders cancers }\end{array}$} & Medium & $1.09(0.67,1.78)$ & $1.10(0.66,1.81)$ & $1.10(0.67,1.83)$ & $1.11(0.67,1.84)$ \\
\hline & Low & 1.00 & 1.00 & 1.00 & 1.00 \\
\hline $\mathrm{p}$ for trend & & 0.71 & 0.73 & 0.71 & 0.73 \\
\hline & High & $1.63(0.77,3.46)$ & $1.67(0.77,3.60)$ & $1.55(0.72,3.34)$ & $1.64(0.75,3.58)$ \\
\hline \multirow{2}{*}{ (42 deaths) } & Medium & $1.35(0.62,2.92)$ & $1.37(0.63,3.02)$ & $1.35(0.62,2.96)$ & $1.37(0.63,3.02)$ \\
\hline & Low & 1.00 & 1.00 & 1.00 & 1.00 \\
\hline $\mathrm{p}$ for trend & & 0.20 & 0.19 & 0.27 & 0.21 \\
\hline & High & $0.97(0.50,1.88)$ & $1.05(0.53,2.06)$ & $0.87(0.44,1.70)$ & $0.91(0.46,1.81)$ \\
\hline \multirow{2}{*}{ (45 deaths) } & Medium & $0.52(0.24,1.14)$ & $0.55(0.25,1.23)$ & $0.54(0.24,1.20)$ & $0.55(0.25,1.24)$ \\
\hline & Low & 1.00 & 1.00 & 1.00 & 1.00 \\
\hline $\mathrm{p}$ for trend & & 0.85 & 0.96 & 0.64 & 0.77 \\
\hline & High & $0.96(0.46,2.25)$ & $1.01(0.45,2.24)$ & $0.97(0.43,2.16)$ & $1.05(0.46,2.39)$ \\
\hline \multirow{2}{*}{ (37 deaths) } & Medium & $0.83(0.38,1.84)$ & $0.87(0.39,1.94)$ & $0.83(0.37,1.86)$ & $0.85(0.38,1.92)$ \\
\hline & Low & 1.00 & 1.00 & 1.00 & 1.00 \\
\hline $\mathrm{p}$ for trend & & 0.91 & 0.98 & 0.93 & 0.92 \\
\hline
\end{tabular}

${ }^{\star}$ Cigarettes smoked daily, ex, current, never smokers, alcohol consumption $(0,>0-15,>15$ units weekly), hours of exercise weekly, diastolic blood pressure (mm Hg), body mass index $\left(\mathrm{kg} / \mathrm{m}^{2}\right), \mathrm{FEV}_{1} \%$. 
Table 6 All cause and cause specific mortality according to the difference between perceived stress at first and second screening

\begin{tabular}{|c|c|c|c|c|c|}
\hline & Perceived stress & Adjusted for age & $\begin{array}{l}\text { Adjusted for age and } \\
\text { occupational class }\end{array}$ & $\begin{array}{l}\text { Adjusted, for age, } \\
\text { occupational class, and risk } \\
\text { factors }\end{array}$ & $\begin{array}{l}\text { Adjusted for age, occupational } \\
\text { class, risk factors and } \\
\text { reporting tendency }\end{array}$ \\
\hline All cause & Increased & $0.97(0.79,1.18)$ & $0.97(0.80,1.19)$ & $0.98(0.80,1.21)$ & $0.99(0.80,1.21)$ \\
\hline \multirow[t]{2}{*}{ (642 deaths) } & Stable & $0.95(0.79,1.15)$ & $0.95(0.79,1.14)$ & $0.99(0.81,1.17)$ & $0.97(0.80,1.16)$ \\
\hline & Decreased & 1.00 & 1.00 & 1.00 & 1.00 \\
\hline $\mathrm{p}$ for trend & & 0.73 & 0.78 & 0.91 & 0.88 \\
\hline All cardiovascular & Increased & $1.04(0.78,1.37)$ & $1.04(0.79,1.38)$ & $1.06(0.80,1.40)$ & $1.05(0.80,1.39)$ \\
\hline \multirow[t]{2}{*}{ (335 deaths) } & Stable & $0.95(0.73,1.22)$ & $0.95(0.73,1.22)$ & $0.97(0.75,1.25)$ & $0.96(0.74,1.23)$ \\
\hline & Decreased & 1.00 & 1.00 & 1.00 & 1.00 \\
\hline $\mathrm{p}$ for trend & & 0.83 & 0.79 & 0.71 & 0.75 \\
\hline Coronary heart disease & Increased & $1.08(0.79,1.48)$ & $1.09(0.80,1.49)$ & $1.11(0.81,1.52)$ & $1.10(0.80,1.51)$ \\
\hline \multirow{2}{*}{ (258 deaths) } & Stable & $0.91(0.68,1.21)$ & $0.91(0.68,1.21)$ & $0.92(0.69,1.24)$ & $0.91(0.68,1.23)$ \\
\hline & Decreased & 1.00 & 1.00 & 1.00 & 1.00 \\
\hline $\mathrm{p}$ for trend & & 0.65 & 0.62 & 0.55 & 0.57 \\
\hline Smoking related cancers & Increased & $0.65(0.40,1.06)$ & $0.66(0.41,1.07)$ & $0.65(0.40,1.06)$ & $0.65(0.40,1.06)$ \\
\hline \multirow[t]{2}{*}{ (126 deaths) } & Stable & $0.87(0.58,1.28)$ & $0.86(0.58,1.28)$ & $0.87(0.58,1.29)$ & $0.86(0.58,1.28)$ \\
\hline & Decreased & 1.00 & 1.00 & 1.00 & 1.00 \\
\hline $\mathrm{p}$ for trend & & 0.09 & 0.09 & 0.09 & 0.08 \\
\hline Other cancers & Increased & $0.86(0.49,1.48)$ & $0.86(0.49,1.49)$ & $0.88(0.51,1.53)$ & $0.88(0.51,1.53)$ \\
\hline \multirow[t]{2}{*}{ (89 deaths) } & Stable & $0.92(0.57,1.49)$ & $0.92(0.57,1.49)$ & $0.93(0.57,1.51)$ & $0.93(0.57,1.50)$ \\
\hline & Decreased & 1.00 & 1.00 & 1.00 & 1.00 \\
\hline $\mathrm{p}$ for trend & & 0.58 & 0.58 & 0.65 & 0.64 \\
\hline Stroke & Increased & $0.91(0.41,2.01)$ & $0.91(0.41,2.01)$ & $0.92(0.42,2.04)$ & $0.92(0.42,2.03)$ \\
\hline \multirow[t]{2}{*}{ (42 deaths) } & Stable & $0.90(0.44,1.82)$ & $0.90(0.44,1.82)$ & $0.91(0.45,1.85)$ & $0.90(0.44,1.83)$ \\
\hline & Decreased & 1.00 & 1.00 & 1.00 & 1.00 \\
\hline $\mathrm{p}$ for trend & & 0.81 & 0.81 & 0.83 & 0.83 \\
\hline Respiratory & Increased & $1.65(0.79,3.45)$ & $1.66(0.79,3.48)$ & $1.99(0.94,4.22)$ & $1.92(0.90,4.08)$ \\
\hline \multirow{2}{*}{ (45 deaths) } & Stable & $0.99(0.47,2.09)$ & $0.99(0.47,2.09)$ & $1.04(0.49,2.21)$ & $0.99(0.46,2.13)$ \\
\hline & Decreased & 1.00 & 1.00 & 1.00 & 1.00 \\
\hline $\mathrm{p}$ for trend & & 0.17 & 0.17 & 0.07 & 0.08 \\
\hline Alcohol related & Increased & $1.10(0.52,2.34)$ & $1.10(0.52,2.35)$ & $1.14(0.53,2.45)$ & $1.13(0.53,2.42)$ \\
\hline \multirow[t]{2}{*}{ (37 deaths) } & Stable & $0.54(0.24,1.21)$ & $0.54(0.24,1.21)$ & $0.58(0.26,1.31)$ & $0.57(0.25,1.29)$ \\
\hline & Decreased & 1.00 & 1.00 & 1.00 & 1.00 \\
\hline $\mathrm{p}$ for trend & & 0.86 & 0.85 & 0.79 & 0.81 \\
\hline
\end{tabular}

${ }^{\star}$ Cigarettes smoked daily, ex, current, never smokers, alcohol consumption $(0,>0-15,>15$ units weekly), hours of exercise weekly, diastolic blood pressure (mm Hg), body mass index $\left(\mathrm{kg} / \mathrm{m}^{2}\right), \mathrm{FEV}_{1} \%$.

stress is strongly related to lower BMI. High cumulative stress at first and second screening (table 2) was strongly related to lower BMI and less strongly to better lung function. Change in stress over the screening interval (table 3) shows no clear relation with established behavioural and physiological risk factors. Change in stress was not related to change in risk factors (not shown).

Table 4 shows relative risk of all cause and cause specific mortality associated with high and medium reported stress (compared with low stress as baseline) at initial screening. The general pattern apparent is of an ostensibly protective relation, particularly in the case of cancers associated with smoking, which is attenuated and weakened on adjustment for social position. Further adjustment for risk factors and reporting tendency has little effect on all estimates. The exception to this pattern is a weak suggestion of an increased relative risk of respiratory mortality and mortality related to alcohol consumption, particularly after adjustment for current occupational class. Adjustment for other indices of life course social position has little additional influence. The estimate is only moderately attenuated on adjustment for risk factors including alcohol consumption and smoking. Adjustment for these risk factors individually did not substantially change this pattern (not shown).

Measures of social position other than current occupational class and age at initiation and inhalation for smokers were not included in models using second screening data.

Table 5, mortality by the sum of stress scores at first and second screening, shows broadly the same pattern as table 4 . There is a weak suggestion of a small protective effect of higher stress in relation to all cause mortality and a slightly stronger suggestion of a larger protective effect with mortality from smoking related cancers. Both these estimates are considerably attenuated on adjustment for current occupational class. There was a weak suggestion of an increased risk of stroke with higher cumulative stress. Adjustment for risk factors and reporting tendency had little influence on any effect estimates most of which were close to the null value. The increased relative risk of respiratory and alcohol related mortality apparent at first screening was not seen in relation to cumulative stress.

These patterns are repeated in table 6 showing mortality by change in stress score. Few of these estimates are markedly different from the null value. Again there is a moderately strong suggestion of a protective effect of increasing stress in relation to smoking related cancers. Conversely, respiratory mortality is positively associated with increasing stress.

\section{Discussion}

It seems unlikely that stress, which is associated with increased smoking, ${ }^{24}$ is protective against smoking related cancers. It is similarly unlikely that the suggestion of a protective effect of higher stress, in relation to all cause and cardiovascular mortality, is genuine. Rather, our results reflect the influence of confounding in observational epidemiology. ${ }^{36}$

The association between adverse lifetime material circumstances and poorer health in this population has been described previously. ${ }^{37}$ The apparent protective relation between higher stress and better health was attributable 
to the association between higher stress and material advantage in this population. The relation was weakened on adjustment for current occupational class, one indicator of material circumstances. Adjustment for additional indices of social position had little additional influence on the model containing current occupational class. Adjustment for established disease risk factors made, in most instances, little difference.

The ability of confounding to generate apparently robust, independent, yet spurious associations in observational epidemiology is well established, ${ }^{38}$ indeed the phenomenon is the mainstay of submissions to the traditionally light hearted Christmas edition of the British Medical fournal. ${ }^{39-41}$ Essentially, all that separates these latter associations from those presented as meaningful is plausibility. Apparent plausibility is, arguably, the weakest criterion for causality. ${ }^{38}$ Plausibility of an association between stress, and other psychosocial exposures, and health is well established. ${ }^{7}$ Nevertheless it is probable that many, apparently plausible associations between psychosocial exposures and health, reported in the literature are as much the product of confounding, and therefore equally spurious, as those we have reported here.

The RSI has been superseded by more elaborate stress measures in some more recent studies. ${ }^{42}$ Conversely, evidence of a positive relation between stress and health has often rested on simpler measures of stress. ${ }^{17}$ The main points of this paper do not relate to the status of the RSI among psychometric instruments (though it appears as reliable as many, ${ }^{42-45}$ and we have argued its validity above). The issues we have highlighted are pertinent to any situation where the construct under study is socially patterned. Indeed the consistent relation between certain psychosocial constructs - those indexing different aspects of personal autonomy for example, ${ }^{46}$ and health seems likely to be attributable to the fact that such constructs are consistently related to social position (even to the extent that they are incorporated as measures of this in contemporary social classifications ${ }^{47}$ ).

It could be argued that our instrument was differentially sensitive to stress in different social groups. Repeating analyses for men in manual and non-manual occupations separately revealed that a "protective" effect was essentially confined to the latter (age adjusted hazard ratio for all cause mortality high versus low stress manual 1.02 (95\% CI 0.83, 1.25), non-manual 0.79 (95\% CI 0.60, 1.02); for smoking-related cancers manual 0.95 (95\% CI $0.59,1.51)$, non-manual 0.29 (95\% CI 0.11 , $0.74)$ ). Some under-reporting of stress may have occurred, suggesting that self report may be problematic in this context. Conversely, more objective measures that ignore the dimension of individual perception and coping, central to all the proposed mechanisms through which stress may influence health, may be inappropriate.

Prevalent disease may increase perception, and hence reporting of stress. Ideally all
KEY POINTS

- Various indicators of "psychosocial adversity", such as increased psychological stress, have been shown to be associated with increased mortality.

- In studies showing these associations, psychosocial adversity was also associated with socioeconomic disadvantage.

- In a population where increased stress was associated with socioeconomic advantage psychosocial adversity appeared to reduce mortality.

- This "effect" was probably the product of socioeconomic confounding.

- Many reported "effects" of psychosocial exposures may be attributable to confounding and of no causal significance.

subjects with significant morbidity at recruitment should have been excluded from mortality analyses. As we did not have complete information on all significant morbidity at recruitment it was not possible to do this. Two considerations are important in this regard. Firstly, a "healthy worker" effect would suggest that the prevalence of significant morbidity would be lower in this cohort than in the general population. Secondly, the effect of prevalent morbidity would be to generate a spurious, positive relation between stress and mortality. The confounded negative relation we found was, therefore, despite this effect. This can be seen in relation to CHD. If prevalent cases of CHD (Rose angina positive, $n=336$ ) are excluded from mortality analyses the confounded "protective" effect of stress is accentuated (age adjusted hazard ratio for all cause mortality medium versus low stress 0.82 (95\% CI $0.73,0.92)$, high versus low stress 0.82 $(95 \%$ CI $0.69,0.97))$.

We have illustrated difficulties caused by confounding, in the interpretation of observed associations between psychosocial exposures and health. We have also shown how the traditional approach to this issue, adjustment for the confounding factor by including a measure of it in multivariate models, may not be fully successful. Specificity of association between exposure and outcome can be sought as evidence against confounding. "Blanket" associations between various unrelated psychosocial exposures and similarly distinct health outcomes are strongly suggestive of confounding. ${ }^{48-50}$ Though interest has focused on psychosocial factors as potential determinants of health inequality it may be more valuable to study them in populations where they are not associated with material adversity as a positive association with health in this circumstance is difficult to attribute to confounding.

It could be argued that reducing the stressfulness of modern life, creating fairer workplaces and generally being nicer to each other are ethical imperatives in little need of further epidemiological justification. ${ }^{51}$ We strongly agree with this, but believe current evidence does not support the suggestion that population health is amenable to improvement 
through psychosocial interventions. Rather, health improvements and reductions in health inequalities are more likely to result from interventions aimed at reducing material disadvantage and at improving behavioural and physiological risk profiles.

The work of Victor Hawthorne, Charles Gillis, David Hole and Pauline MacKinnon has provided us with the data required for this analysis.

Funding: this work was supported by a grant within phase two of the Health Variations research programme of the Economic and Social Research Council. Preliminary analyses on this data were undertaken by JM while he was supported by a clinical epidemiology training fellowship from the Wellcome Trust. Conflicts of interest: none.

1 Davison C, Davey Smith G, Frankel SJ. Lay epidemiology and the prevention paradox: the implications of the coronary candidate for health promotion. Sociol Health Illn 1991;13:1-19.

2 Greenwood DC, Muir KR, Packham CJ, et al. Coronary heart disease: a review of the role of psychosocial stress and social support. F Public Health Med 1996;18:221-31

3 Hemingway $H$, Marmot $M$. Evidence based cardiology: Psychosocial factors in the aetiology and prognosis of coronary heart disease: systematic review of prospective cohort studies. BMF 1999;318:1460-7. 4 Syme SL, Balfour JL. Explaining inequalities in coronary

5 Marmot MG Improvement of social environment to improve health. Lancet 1998;351:57-60.

6 Shively CA, Clarkson TB. Social status and coronary artery atherosclerosis in female monkeys. Arterioscler Thromb 1994;14:721-6.

7 Brunner E. Stress and the biology of inequality. BMF 1997; 314:1472-6.

8 Schachter S. Silverstein B. Kozlowski LT, et al. Effects of stress on cigarette smoking and urinary $\mathrm{pH}$. F Exp Psychol Gen 1977;106:24-30.

9 Hellerstedt WL, Jeffery RW. The association of job strain and health behaviours in men and women. Int 7 Epidemio 1997;26:575-83.

10 Watson D, Pennebaker JW. Health complaints, stress and distress: exploring the central role of negative affectivity. Psychol Rev 1989;96:234-54.

11 Bosma H. Marmot MG. Hemingway H, et al. Low job control and risk of coronary heart disease in Whitehall II (prospective cohort) study. BMJ 1997;314:558-65.

12 Marmot MG. Bosma H. Hemingway $\mathrm{H}$, et al. Contribution of job control and other risk factors to social variations in coronary heart disease incidence. Lancet. 1997;350:235-9.

13 Bosma H, van de Mheen HD, Mackenbach JP. Social class in childhood and general health in adulthood: questionnaire study of contribution of psychological attributes. BMF 1999;318:18-22.

14 Cheng Y, Kawachi I, Coakley EH, et al. Association between psychosocial work characteristics and health functioning in American women: prospective study. BMf 2000;320:1432-

15 Vahtera J, Kivimaki M, Pentti J, et al. Effect of change in the psychosocial work environment on sickness absence: a seven year follow up of initially healthy employees. F Epidemiol Community Health 2000;54:484-93.

16 Macleod J. Davey Smith G, Heslop P, et al. Always look on the bright side of life? The influence of reporting tendency when exposure and outcome measurements are based on self -report. [Abstract]. 7 Epidemiol Community Health self -report.

17 Rosengren A, Tibblin G, Wilhelmsen L. Self-perceived psychological stress and incidence of coronary artery disease in middle-aged men. Am f Cardiol 1991;68:1171-5.

18 Bosma H, Schrijvers C, Mackenbach JP. Socioeconomic inequalities in mortality and importance of perceived control: cohort study. BMF 1999;319:1469-70.

19 Orth-Gomer K, Wamala SP, Horsten M, et al. Marital stress worsens prognosis in women with coronary heart disease.
The Stockholm Female Coronary Risk Study. $\mathscr{F} A M A$ 2000;284:3008-14

20 Haines A, Cooper J, Meade TW. Psychological characteristics and fatal ischaemic heart disease. Heart 2001;85:3859.

21 Davey Smith G, Harding S. Is control at work the key to socio-economic gradients in mortality? Lancet 1997;350: 1369-70.

22 Macleod J Mortality amongst elderly caregivers. $\mathcal{F} A M A$ 2000;283:2105-6.
23 Wainwright D, Calnan M. Rethinking the work stress "epidemic”. Eur fै Public Health 2000;10:231-4.

24 Heslop P, Davey Smith G, Carroll D, et al. Perceived stress and coronary heart disease risk-factors: The contribution of socio-economic position. British fournal of Health Psychology 2001;6:167-8.

25 Reeder LG, Chapman JM, Coulson AH. Socioenvironmental stress, tranquilizers and cardiovascular disease. Proceedings of the Excerpta Medica International Congress Series 1968;182:226-38.

26 Reeder LG, Schrama PGM, Dirken JM. Stress and cardiovascular health: an international cooperative study-I. Soc Sci Med 1973;7:573-84.

27 Haynes SG, Levine S, Scotch N, et al. The relationship of psychosocial factors to coronary heart disease in the Framingham Study: Methods and risk factors. Am $\mathcal{F}$ Epidemiol 1978;107:362-83.

28 Chapman JM, Reeder LG, Massey J, et al. Relationships of stress, tranquilizers and plasma cholesterol levels in a sample population under study for coronary heart disease. $\mathrm{Am}$ f Epidemiol 1966;83:537-47.

29 Davey Smith G, Hart C, Hole D, et al. Education and occupational social class: which is the more important indicator of mortality risk? f Epidemiol Community Health 1998;52: 153-60.

30 OPCS. Classification of occupations 1966. London: HMSO, 1966.

31 Carstairs V, Morris R. Deprivation and health in Scotland. Aberdeen: Aberdeen University Press, 1991.

32 Davey Smith G, Shipley M, Leon DA. Height and mortality from cancer among men: prospective observational study. BMF 1998;317:1351-2.

33 Hart CL, Davey Smith G, Hole DJ, et al. Alcohol consumption and mortality from all causes, coronary heart disease, and stroke: results from a prospective cohort study of Scottish men with 21 years of follow up. BMF 1999;318:1725-

34 Cox DR. Regression models and life tables. Fournal of the Royal Statistical Society Series B 1972;34:187-220.

35 Stata Corporation. Stata statistical software: release 6.0. College Station, Houston, TX: Stata Corp, 1998.

36 Davey Smith G, Phillips AN. Confounding in epidemiological studies: why "independent" effects may not be all they seem. BM7 1992;305:757-9.

37 Davey Smith G, Hart C, Blane D, et al. Lifetime socioeconomic position and mortality: prospective observational study. BMF 1997;314:547-52.

38 Davey Smith G, Phillips AN, Neaton JD. Smoking as "independent" risk factor for suicide: illustration of an artifact from observational epidemiology? Lancet 1992;340:70912

39 Bygren LO, Konlaan BB, Johansson S. Attendance at cultural events, reading books or periodicals, and making music or singing in a choir as determinants for survival: Swedish interview survey of living conditions. BMF 1996;313:1577-80.

40 Davey Smith G, Dorling D. "I'm all right John": voting patterns and mortality in England and Wales, 1981-92. BMF 1996;313:1573-7.

41 Davey Smith G, Frankel S, Yarnell J. Sex and death: are they related? Findings from the Caerphilly cohort study. $B M F$ 1997;315:1641-4

42 Cohen S, Kamarck T, Mermelstein R. A global measure of perceived stress. F Health Soc Behav 1983;24:385-96.

43 Alterman T, Shekelle RB, Vernon SW, et al. Decision latitude, psychologic demand, job strain and coronary heart disease in the Western Electric Study. Am F Epidemiol 1994;139:620-7.

44 Yarnold PR, Mueser KT. Meta-analyses of the reliability of Type A behaviour measures. Br f Med Psychol 1989;62:4350

45 Goldberg G. General Health Questionnaire (GHQ-12). Windsor: NFER-Nelson. 1992

46 Marmot M, Bobak M. International comparators and poverty and health in Europe. BMF 2000;321:1124-8.

47 Rose D, O'Reilly K. Final report of the ESRC review of government and social classifications. Swindon: Economic government and social classifications. Swindon: Economic Statistics, 1998

48 Kauhanen J, Kaplan GA, Cohen RD, et al. Alexthymia and risk of death in middle-aged men. $\mathcal{F}$ Psychosom Res 1996;41: $541-9$

49 Everson SA, Kauhanen J, Kaplan GA, et al. Hostility and increased risk of mortality and acute myocardial infarction: the mediating role of behavioural risk factors. Am $\mathcal{F}$ Epidemiol 1997; 146:142-52.

50 Everson SA, Goldberg DE, Kaplan GA, et al. Hopelessness and risk of mortality and incidence of myocardial infarction and cancer. Psychosom Med 1996;58:113-21.

51 Hemingway $\mathrm{H}$, Marmot $M$. Relation between hostility and coronary heart disease - evidence does not support a link (authors' reply). BMF 1999;319:917. 